\title{
Religion, spirituality and neurorehabilitation
}

\author{
Simon Dein \\ Derwent Centre, Princess Alexandra Hospital, Hamstel Road, Harlow, CM20 1QX, UK \\ Tel.: +44 01279637106; E-mail: s.dein@ucl.ac.uk
}

\section{Introduction}

This thematic section in Neurorehabilitation focuses upon spiritual and religious aspects of the neurorehabilitation process. Beyond their direct impact on the brain, neurological insults affect the sense of self, personhood and identity. Cultural and religious factors determine how the injury is expressed, the response of the patient and his or her family, interpersonal relationships but also the relationship with medical professionals and the outcomes of neurorehabilitation.

It is necessary to distinguish between religion which is institutionalised, community based, associated with doctrine, beliefs, practices and rituals from spiritualty which is an individual relationship with a higher power. Although the phenomena are related, being spiritual does not necessarily imply being religious whereas the reverse is generally true.

As Redfern and Coles (2015) note, the relationship between religiosity, spirituality and neurological disorders needs to differentiate between intrinsic loss of religiosity directly resulting from neural degeneration; the contingent reduction in spirituality with the practical social and cognitive effects brought about by the disease and any reactive response subsequent to developing the disease which might enhance or diminish religious faith. These authors point out how neurological disorders often lead to major upheavals in terms of lifestyle, future plans and future expectations and prompt existential questions pertaining to the meaning and purpose of life. Patients may frequently ask 'why me?' related questions and their suffering can be exacerbated by the spiritual pain they experience.

\section{Existing literature}

To date there has been rather limited research specifically addressing spirituality and neurodisability and more specifically its implications for enhancing neurorehabilitation. Previous literature has focused upon the impact of spirituality on a number of neurological disorders including traumatic brain injury (Waldron Perrine et al. 2011; Jones, Pryor, Care-Unger \& Simpson 2018), post traumatic growth after head injury (Grace et al. 2015; Rogan et al. 2013), Parkinson's disease (Reynolds 2017; Redfern \& Coles 2015), Motor Neurone Disease (Macleod \& Clarke 2007; Simmons 2013; Cheng et al. 2017), spinal cord injury (Jones, Dorset, Simpson \& Briggs 2018), stroke (Giaquinto, Spiridigliozzi \& Caracciolo 2007; Laures Gore 2018) and epilepsy (Tedrus, Fonseka \& Hoeahr 2014; Rigon et al. 2019).

An overview of the extant literature suggests that spiritual coping impacts positively on adjustment, mental health, quality of life and possibly physical outcomes in a range of neurological disorders. In general higher levels of spirituality are associated with better measures of mental health and to a lesser extent physical health in those with neurodisability. It appears that there are small positive correlations between spiritual interventions and health in neurorehabilitation populations but this finding may directly result from methodological issues e.g. patients pray- 
ing both as health deteriorates and as it improves (Lomay \& Johnstone 2015).

\section{The current edition}

Written by specialists in their field, the papers included here make important contributions to the emerging and clinically significant literature on spirituality and neurorodisability and provide pertinent suggestions for incorporating spirituality into neurorehabilitation. Simpson, Anderson, Jones, Genders and Gopinath use a structural equation modelling approach to examine whether spirituality, resilience and hope mediate outcomes among family caregivers after traumatic brain injury or spinal cord injury. Their findings demonstrate that the proposed model is a good fit and they provide important data for planning family based interventions in these disorders. Jones, Pryor, Care-Unger and Simpson report on an online survey of rehabilitation professionals' perceptions of spirituality and spiritual care. The results indicate that while spirituality is considered to play a significant role in rehabilitation after traumatic brain injury or spinal cord injury, most staff felt poorly equipped to provide spiritual care.

In a scoping review, Gibbs Anderson, Simpson and Jones focus upon spirituality and resilience among family caregivers of stroke survivors. The authors find that spirituality and resilience are significant factors in caregiver adjustment following stroke. Finally in relation to Parkinson's Disease, Redfern, Mason, Barker and Coles assess the claim that Parkinson's Disease reduces religious faith and spirituality in those who suffer with this disease. The study indicates that compared to non-neurological patients with similar levels of disability, Parkinson's disease does not result in a decline in religious faith or spirituality and furthermore declining mobility and cognition in this disorder does not result in diminished religiosity.

\section{References}

Giaquinto, S., Spiridigliozzi, C., \& Caracciolo, B. (2007). Can faith protect from emotional distress after stroke? Stroke, 38(3), 993-997.

Grace, J. J., Kinsella, E. L., Muldoon, O. T., \& Fortune, D. G. (2015). Post-traumatic growth following acquired brain injury: A systematic review and meta-analysis. Front Psychol, 6, 1162.

Lomay, V. \& Johnstone, B. (2015). Spirituality, Religiousness, and Culture in Neurorehabilitation in Uomoto J.M. (ed) Multicultural Neurorehabilitation Clinical Principals for Rehabilitation Professionals. Springer Publishing Company.

Jones, K. F., Dorsett, P., Simpson, G. \& Briggs, L. (2018). Moving forward on the journey: Spirituality and family resilience after spinal cord injury. Rehabilitation Psychology, 63(4), 521-531.

Jones, K. Pryor, J., Care-Unger C., \& Simpson, G. (2018). Spirituality and its relationship with positive adjustment following traumatic brain injury: A scoping review. Brain Injury, 32, 13-14, 1612-1622.

Karagiorgou, O., Evans, J., \& Cullen, B. (2018). Post-traumatic growth in adult survivors of brain injury: A qualitative study of participants completing a pilot trial of brief positive psychotherapy. Disability and Rehabilitation, 40(6), 655-659.

Laures-Gore, J. S., Lambert, P. L., Kruger, A. C. et al. (2018). Spirituality and post stroke aphasia recovery. J Relig Health, 57, 1876

McLeod, J. E., \& Clarke, D. M., (2007). A review of psychosocial aspects of motor neurone disease. J Neurol Sci 258, 4-10.

Redfern, C., \& Coles, A. (2015). Parkinson's disease, religion and spirituality. Movement Disorders Clinical Practice, 2, 341346.

Reynolds, D. (2017). Spirituality as a coping mechanism for individuals with Parkinson's Disease. J Christ Nurs, 34(3), 190-194.

Rogan, C., Fortune, D. G., \& Prentice, G. (2013). Post-Traumatic Growth, Illness Perceptions and Coping in People with Acquired Brain Injury. Neuropsychological Rehabilitation, 23(5), 639-657.

Simmons, Z. (2013). Rehabilitation of motor neuron disease. Handb Clin Neurol, 110, 483-498.

Tedrus, G. M., Fonseca, L. C., \& Höehr, G. C. (2014). Spirituality aspects in patients with epilepsy. Seizure, 23(1), 25-28.

Waldron-Perrine, B., Rapport, L. J., Hanks, R. A., Lumley, M., Meachen, S.-J., \& Hubbarth, P. (2011). Religion and spirituality in rehabilitation outcomes among individuals with traumatic brain injury. Rehabilitation Psychology, 56(2), 107116. 